\title{
Aspergillus epidural abscess in a patient with obstructive airway disease
}

\author{
Y. C. CHEE \\ M.B., B.S.(Singapore), M.R.C.P. (U.K.), \\ M.Med(Int.Med) (Singapore) \\ S. C. POH \\ M.B., B.S.(Malaya), F.R.C.P.(E)
}

Department of Medicine III, Tan Tock Seng Hospital, Moulmein Road, Singapore 1130

\begin{abstract}
Summary
A 54-year-old Chinese man with episodic bronchial asthma since 25 years of age was treated for pulmonary tuberculosis in 1976 because of left upper lobe lesions on chest radiograph. In 1981 he presented with an extradural mass compressing the thoracic spinal cord, thought to be tuberculosis but which on biopsy was found to be aspergillosis. Sputum culture, type one skin-prick reactivity and serum precipitating antibodies were positive for Aspergillus. Amphotericin B intravenously, then ketoconazole orally did not substantially improve his clinical course. He died about four months postlaminectomy.
\end{abstract}

KEY WORDS: aspergillosis, asthma, tuberculosis, epidural abscess.

\section{Introduction}

Aspergillus is an ubiquitous saprophytic fungus. Few species are pathogenetic to humans. The infection usually involves the lungs, skin, external ears, paranasal sinuses, orbits and occasionally the bones and meninges. The central nervous system becomes infected either by haematogenous spread from the lungs or gastrointestinal tract (Young et al., 1970) or by direct invasion from the paranasal sinuses or orbit (Warder, Chikes and Hudson, 1975; Mukoyama, Gimple and Poser, 1969). Central nervous system or systemic aspergillosis almost always occurs on a background of underlying neoplastic disease, collagen-vascular disease or iatrogenic immunosuppression. Epidural abscess as a manifestation of aspergillosis is rare. Ingwer, McLeish and Tight (1978) reported such a case in a renal transplant recipient; we report such a presentation in a patient with obstructive airway disease not receiving corticosteroids.

\section{Case report}

In August 1981, a 54-year-old Chinese man was admitted to this hospital with progressive weakness, over one week, of both legs with corresponding loss of sensation. He had been asthmatic since the age of 25 years. Attacks of asthma were episodic with symptom-free intervals and he did not need maintenance therapy. He was a storekeeper and never smoked cigarettes. In 1976, he was diagnosed as having pulmonary tuberculosis and was treated for one year by his private practitioner. He was never on corticosteroid therapy.

In hospital, he gave a history of pain at the back of his left chest and over the thoracic spine lasting four months. It was increasing in severity and one week before admission, he developed neurological deficits in his legs. He had weight loss, cough with purulent sputum and for the last five years had bouts of haemoptysis.

Physical examination showed a febrile thin man with normal vital signs. Examination of the lungs was clear; the heart and abdomen were unremarkable. Neurological testing showed normal cranial nerves and upper limbs. There was decreased sensation to pin-prick below the fifth thoracic dermatome level. Bladder and bowel sphincters were normal. The left lower limb was weaker than the right, had an extensor plantar response and showed decreased proprioceptive sensation. Lower limb reflexes were hyperreactive. No spinal tenderness was elicited.

A chest radiograph showed marked pleural thickening in the left upper zone with fibrotic changes in both upper and mid zones consistent with old tubercular changes. Lung fields were slightly hyperinflated. Radiographs of the thoracic spine were normal.

Laboratory evaluation included a peripheral white blood cell count of $8.7 \times 10^{4} /$ litre with no eosinophilia, a haemoglobin of $10.7 \mathrm{~g} / \mathrm{dl}$, normal serum creatinine and electrolytes and an elevated erythrocyte sedimentation rate of $129 \mathrm{~mm}$ in the first hour. Lumbar puncture revealed xanthochromic fluid at normal pressure with 7 cells, a glucose level of 1.78 
$\mathrm{mmol} / \mathrm{litre}$, a protein level of $4 \mathrm{~g} /$ litre and the presence of globulin. There were a few lymphocytes seen on the smear but no torula, acid-fast bacilli or other organisms. His arterial blood gas was normal.

In view of his previous tuberculosis history, he was started on second line anti-tuberculous chemotherapy (kanamycin, ethambutol, isoniazid, rifampicin and pyrazinamide) as for tuberculosis of the spine. A lumbar myelogram showed a complete block to the flow of dye at the fourth thoracic vertebra level due to extradural compression. Laminectomy of the third and fourth thoracic vertebrae revealed epidural granulomatous tissue densely adherent to the dura compressing the spinal cord at that level but not infiltrating it. The vertebrae were not involved.

Histological staining of the tissue obtained revealed branching septate organisms that resembled Aspergillus. Subsequently this was grown on sputum culture. Precipitins to Aspergillus were present in the blood. Skin-prick test for immediate hypersensitivity using Bencard Testing Solutions Group M2 moulds (Aspergilli) was positive at $1 \mathrm{~mm}$ weal. His Mantoux test was negative using one tuberculin unit of purified protein derivative. His peripheral blood had a normal proportion of $B$ and $T$ cells and normal immunoglobulin levels.

Therapy with amphotericin B was poorly tolerated and only $231 \mathrm{mg}$ were given intravenously in addition to the antituberculous therapy. He developed severe reversible liver and renal impairment. The neurologic deficits did not improve. When he recovered from the toxicity of amphotericin, he was started on oral ketoconazole $200 \mathrm{mg}$ daily (on the 63rd postoperative day) but he died after a further 56 days (total dose of ketoconazole was $1120 \mathrm{mg}$ ) of respiratory failure due to his chronic lung disease and the complicating paraplegia.

\section{Discussion}

Central nervous system involvement may occur in patients with disseminated aspergillosis but epidural abscess is very rare (Shaw and Warthen, 1936; Wybel, 1952; Hughes, 1965; Seres, Ono and Benner, 1972; Ingwer et al., 1978). Such patients are usually immunocompromised. The immunosuppressed renal transplant recipient with epidural Aspergillus fumigatus abscess reported by Ingwer and his colleagues was exposed to three factors that increased the risk of developing fungal infections. These were treatment with immunosuppressive agents, broad-spectrum antibiotic therapy and acute transplant rejection necessitating the exhibition of repeated courses of high dose corticosteroids. Our patient had none of these factors and was never on long-term corticosteroids for his asthma.
Aspergillus species occur as a commensal in many as $10 \%$ of random sputum samples and in such instances, no host response occurs. All immunological tests would be negative (Turner-Warwick, 1979). The weak positive skin-prick response to Aspergilkws makes it unlikely that Aspergillus is responsible for his asthma as it has been shown that weals of $3 \mathrm{~mm} \overline{\bar{q}}$ greater are more likely to correlate with positige bronchial provocation testing to that allergen than smaller weals (Bryant, Burns and Lazarus, 1975). The absence of both blood eosinophilia and transie radiographic shadows rules out bronchopulmonary aspergillosis.

This patient's chest radiograph showed bilategh contracted upper lobes very suggestive of pulmona tuberculosis for which he received treatment, but the same could be the chronic consequence of repeateg Aspergillus infection as his sputum cultures wefe positive for Aspergillus. The negative Mantoux reastion could result from depressed delayed hypersenș tivity. Aspergillosis accounts for the presence ef precipitins in his blood to Aspergillus.

A primary pulmonary or gastrointestinal focณ̂s with subsequent local or haematogenous spread is the most frequent route of dissemination for the orga ism (Young et al., 1970). Our patient probably hadta pulmonary source with metastatic localization ipte epidural space. Even in the presence of meningitis smear and culture for Aspergillus in cerebrospina fluid is rarely positive (Young et al., 1970). The most reliable method of diagnosis remains biopsy wi histological examination and culture. Unfortunatele the culture was negative perhaps because of pretreas ment of the specimen with sodium hydroxide sol $\overrightarrow{\mathrm{g}}$ tion before culture for mycobacteria.

Amphotericin B has not offered effective chemo therapy for systemic or central nervous system aspergillosis (Young et al., 1970; Bennett, 1974; Utizz, 1974). Recently, an oral antifungal agent, ketocon zole, was found to be efficacious in systemic fung? infections in animal models. The initial clinicg evaluation in man was also promising with no toxicity noted (Borelli et al., 1979). At the recom mended dosage of $200 \mathrm{mg}$ daily, the present patien was given a total of 56 days therapy. It is difficult te evaluate the value of the therapy. The paraplegia remained static probably secondary to vasculat thrombosis (Young et al., 1970). The fourth thoracic vertebra level is usually the anatomical watershed zone for the spinal cord's blood supply, and thereforo an easily compromised situation.

\section{References}

BENNETT, J.E. (1974) Chemotherapy of systemic mycoses. Nex England Journal of Medicine, 289, 30, 320.

Borelli, D., Fuentes, J., Leiderman, E. Restrapo, M.A., Bra J.L., Legendre, R., Levine, H.B. \& Stevens, D.A. (1979)

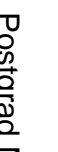


Ketoconazole: an oral antifungal: laboratory and clinical assessment of imidazole drugs. Postgraduate Medical Journal, 55, 657.

BRYANT, D.J., BURNS, M.W. \& LAZARUS, L. (1975) The correlation between skin tests, bronchial provocation tests and the serum level of $\operatorname{lgE}$ specific for common allergens in patients with asthma. Clinical Allergy, 5, 145.

HUGHES, W.T. (1965) Generalized aspergillosis. American Journal of Diseases of Children, 112, 262.

INGWER, I., MCLEISH, K.R. \& TIGHT, R.R. (1978) Apergillus fumigatus epidural abscess in a renal transplant recipient. Archives of Internal Medicine, 138, 153.

Mukoyama, M., Gimple, K. \& Poser, C.M. (1969) Aspergillosis of the central nervous system. Report of brain abscess due to Aspergillus fumigatus and review of the literature. Neurology (Minneapolis), 19, 967.

Seres, S.L., ONo, H. \& BenNeR, E.J. (1972) Aspergillosis presenting as spinal cord compression. Journal of Neurosurgery, 36, 221.
Shaw, F.W. \& WARThEN, H.J. (1936) Aspergillosis of bone. Southern Medical Journal, 29, 1070.

TURNER-WARWICK, M. (1979) Aspergillus fumigatus and lung disease. Postgraduate Medical Journal, 55, 642.

UTIZ, J. (1974) Current and future chemotherapy of central nervous system fungal infections. In: Infectious Diseases of the Central Nervous System. Advances in Neurology (Ed by Thompson, R.A. \& Green, J.R.), Vol. 6, pp. 127-132. Raven Press, New York.

WARDER, F.R.. ChIKES, P.G. \& HudSON, W.R. (1975) Aspergillosis of the paranasal sinuses. Archives of Otolaryngology, 101, 683.

WYBEL, R.E. (1952) Mycosis of cervical spinal cord following intrathecal penicillin therapy. Archives of Pathology, 53, 167.

Young, R.C., BenNeTt, J.E., VoGel, C.L. \& DEVITA, V.T. (1970) Aspergillosis: The spectrum of disease in 98 patients. Medicine (Baltimore), 49, 147.

(Accepted 11 May 1982) 